\title{
Characterization of silicon photodiodes for diffuse reflectance signal extraction
}

\author{
S. Pimenta, J. P. Carmo, R. G. Correia, G. Minas \\ Department of Industrial Electronics, University of Minho, \\ Campus de Azurém, Guimarães, Portugal \\ id4474@alunos.uminho.pt, jcarmo@dei.uminho.pt, \\ id3506@alunos.uminho.pt,gminas@dei.uminho.pt
}

\author{
E. M. S. Castanheira \\ Centre of Physics (CFUM), University of Minho, Campus \\ de Gualtar, Braga, Portugal \\ ecoutinho@fisica.uminho.pt
}

\begin{abstract}
Early detection of gastrointestinal (GI) cancers is one of most important concerns in medical field. Its detection at the earliest stage is crucial to increase the patient survival chances. Optical signals extraction and analysis, specifically diffuse reflectance and intrinsic fluorescence, may improve the ability to detect GI dysplasia, once some morphological and biochemical changes on the tissues (related with early cancer progression) can modify these signals' shape and intensity. The project under this paper aims to develop a chip-sized spectroscopy microsystem for the early detection of GI cancer. This paper presents the characterization of silicon photodiodes (n+/p-epilayer type) fabricated in a standard $0.7 \mu \mathrm{m}$ CMOS process, with different dimensions. The main goal is to conclude which photodiode must be fabricated for the microsystem implementation, taking into account its capacity to extract the signals in the relevant spectral band $(350 \mathrm{~nm}-750 \mathrm{~nm})$, not comprising the microsystem dimensions. With this study, it can be concluded that the suitable photodiodes for the detection of those spectroscopic signals are the ones with $125 \times 125 \mu^{2}$ or $100 \times 100 \mu \mathrm{m}^{2}$ active area, once their quantum efficiency varies between $20 \%$ and $55 \%$, approximately, above $450 \mathrm{~nm}$. In spite of the low photodiodes quantum efficiency (lower than $20 \%$ ) below $450 \mathrm{~nm}$, the viability of using the $100 \times 100 \mu^{2}$ photodiode to extract the diffuse reflectance signal, between $350 \mathrm{~nm}$ and $750 \mathrm{~nm}$, was proven using a test phantom representative of a GI tissue.
\end{abstract}

Index Terms- gastrointestinal cancer, spectroscopic signals extraction, on chip integration, photodiodes characterization, quantum efficiency.

\section{INTRODUCTION}

Gastrointestinal (GI) cancer is one of most common cause of cancer-related death over the world (1). Its detection at the early stage, named dysplastic stage, is crucial in order to increase the survival rate (2). However, this early detection is difficult to perform taking into account the conventional visual inspection techniques - endoscopy and colonoscopy - due to the lack of macroscopically easily visible changes on the tissues surface in the dysplastic stage $(2,3)$.

Thus, the only way to detect dysplasia is performing a large number of biopsies, which are invasive, affected by sample errors and the results are not immediately available, leading to a delay on patient's treatment (3).
For all the reasons described above, the development of new methods or tools to an early GI cancer detection have been arising. Most of them are based on spectroscopic techniques. In fact, spectroscopic signals extraction and analysis may improve the ability to detect GI dysplasia, since some morphological and biochemical changes on the tissues can modify these signals' shape and intensity $(4,5)$.

The extraction of the diffuse reflectance signal of a tissue can be used to detect small changes related with cancer progression, since its intensity and shape is affected by absorption and scattering effects. As a result, an increase of hemoglobin, associated with angiogenesis during cancer progression, results in a reduction of the diffuse reflectance signal. Moreover, as the dysplasia is progressing to cancer, the epithelial tissue thickness increases which reduces the quantity of light that reaches the deeper tissues. Thus, less quantity of light reaches the collagen fibers (the main tissue scatterer) in the connective tissue, decreasing scattering and, consequently, the diffuse reflectance signal intensity $(3,5)$.

The extraction of the intrinsic fluorescence signal is also important, since GI tissue has multiple fluorophores related with cell structure and metabolism - collagen and NADH (reduced form of nicotinamide adenine dinucleotide) - working as cancer biomarkers. A decrease of collagen concentration and an increase of NADH concentration is associated with early cancer progression, resulting in a change of the intensity and shape of the fluorescence signal (5).

Several authors have tried to develop systems for spectroscopy signals extraction and detection of GI dysplasia (2, 6-9). However, most of the authors use complex and bulky spectroscopy systems such as: xenon lamps, UV lasers, monochromators, optical fibers and high quantum efficiency detectors. As a result, these systems cannot be integrated with endoscopic equipment. Other authors $(4,10)$ have tried to miniaturize their systems, replacing some of the components with photodiodes or Light Emitting Diodes (LEDs). However, they still use some macroscopic equipment.

Taking advantage of the microtechnologies powerful tool system on chip integration, the development of a spectroscopy microsystem on a chip, without the need of regular optical fibers, monochromators or high quantum efficiency detectors, that can be used in-loco, will have a high clinical value. This is 
the main innovation of the project under this paper. The microsystem can be integrated with the conventional endoscopic equipment and be used as an auxiliary in early GI dysplasia detection. Moreover, the microsystem can also be used as a portable system in a surgery room, for inspecting total removing of the cancerous tissue during surgery.

The microsystem will extract two optical signals - diffuse reflectance and fluorescence - to detect GI dysplasia. To that purpose, a matrix of thin-film optical filters deposited on the top of silicon photodiodes will be fabricated and used. The optical filters will be centered in a set of spectral bands considered relevant to select/extract the spectroscopic signals $(11,12)$. Moreover, miniaturized LEDs will be incorporated on the chip, as illumination sources for diffuse reflectance and fluorescence measurements. Finally, it will be also necessary readout electronics and a wireless mode, all integrated in a single chip, allowing data transmission and its analysis in a computer.

The analysis of the extracted spectroscopic signals (by the implementation of mathematical models $(13,14))$ will be crucial to relate changes in their intensity and shape with GI cancer progression.

This paper focuses on the characterization of silicon photodiodes fabricated in a standard $0.7 \mu \mathrm{m}$ CMOS process, with different dimensions. The main goal is to conclude which photodiode must be fabricated for the microsystem implementation, taking into account their capacity to extract the signals between the relevant spectral bands $(350 \mathrm{~nm}-750 \mathrm{~nm})$, not comprising the microsystem's dimensions.

\section{RESEARCH TEAM}

The research has been developed in the Department of Industrial Electronics and in the Center of Physics, both from University of Minho. The research team is focused on the development of devices for biomedical applications, especially with integrated electronic circuits and optical sensors for analyzing biological tissues and fluids. Information about the research group can be found in:

http://www.dei.uminho.pt/ gminas/AreasInv_en.htm.

\section{PHOTODIODES FABRICATION AND CHARACTERIZATION}

A standard CMOS process allows the fabrication of three types of photodiodes - n-well/p-epilayer, $\mathrm{p}+/ \mathrm{n}$ well and $\mathrm{n}+/ \mathrm{p}$-epilayer. The operating principle is very similar for all of them. However, they differ in their junction depth, which confers different spectral responses (15).

In the visible spectrum range, the light is more efficiently collected by a shallower junction $n+/ p$-epilayer (15). Since this structure provides the best possible quantum efficiency in the desired spectral band $(350 \mathrm{~nm}-750 \mathrm{~nm})$, a set of photodiodes $(\mathrm{n}+/ \mathrm{p}$-epilayer type $)$ with different dimensions $\left(125 \times 125 \mu \mathrm{m}^{2}\right.$, $100 \times 100 \mu \mathrm{m}^{2}$ and $50 \times 50 \mu \mathrm{m}^{2}$ ) were fabricated in a standard $0.7 \mu \mathrm{m}$ n-well CMOS process.

In order to conclude which photodiode is more suitable for spectroscopic signals extraction, the spectral response of each fabricated photodiode was measured, using a commercial calibrated photodiode as reference (Hamamatsu S1336-5BQ).

The experimental setup used for the photodiodes characterization is shown in Fig. 1. It comprises a $250 \mathrm{~W}$ quartz tungsten halogen lamp, as light source; a monochromator (Newport Corner Stone 74125); an optical fiber (Newport Standard Grade FS Fiber Optic), used to direct the light into the box that contains the photodiodes; and a picoammeter (Keithley 487) to measure the photodiodes current.

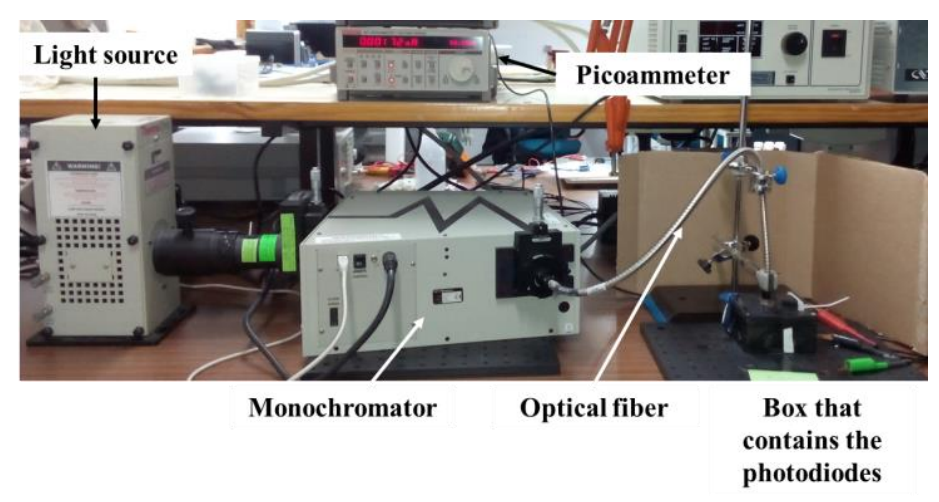

Fig. 1. Experimental setup used for photodiodes characterization.

\section{RESULS AND DISCUSSION}

Figures 2 and 3 show the measured spectral response and the quantum efficiency curves for each fabricated photodiode, respectively.

The quantum efficiency $(\eta(\lambda))$ was obtained from their responsivity $(R(\lambda))$, by the application of equation (1) (16):

$$
\eta(\lambda)=\frac{R(\lambda) h c}{q \lambda}
$$

where $h$ is the Planck constant; $c$ is the velocity of light in a vacuum; and $\lambda$ is the light wavelength.

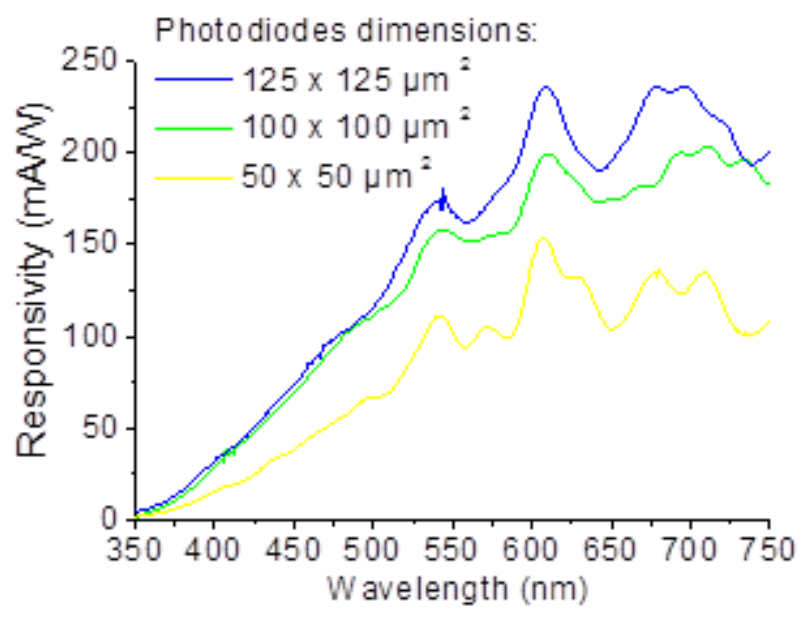

Fig. 2. Measured spectral response of the fabricated photodiodes with different dimensions. 


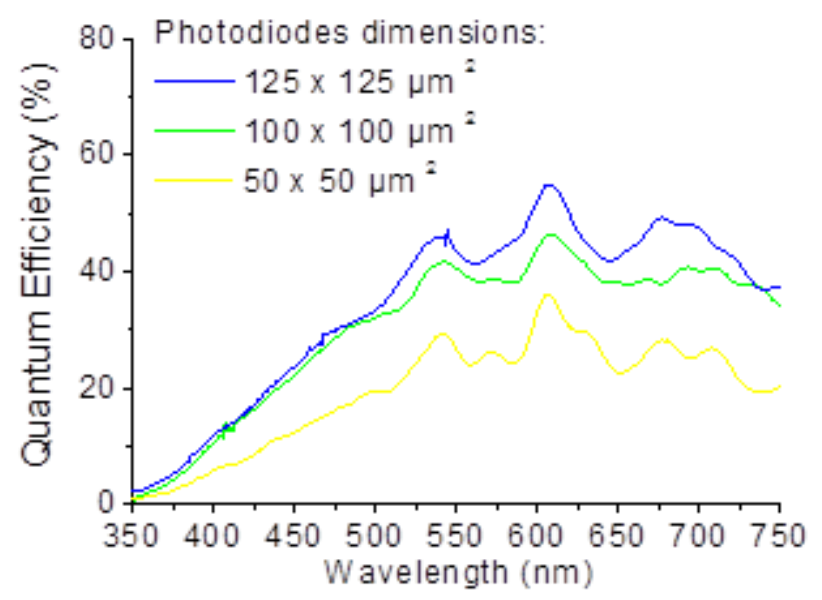

Fig. 3. Measured quantum efficiency of the fabricated photodiodes with different dimensions.

As it can be seen in Fig. 2 and Fig. 3, the use of the CMOS fabricated photodiodes with $125 \times 125 \mu \mathrm{m}^{2}$ or $100 \times 100 \mu \mathrm{m}^{2}$ is suitable to the future implementation of the microsystem and detection of spectroscopic signals, especially between $450 \mathrm{~nm}$ and $750 \mathrm{~nm}$, since their quantum efficiency varies between $20 \%$ and $55 \%$, approximately.

In spite of the low photodiodes quantum efficiency (lower than 20\%) below $450 \mathrm{~nm}$, it was decided to evaluate the viability of using the fabricated photodiode with $100 \times 100 \mu \mathrm{m}^{2}$ to extract a spectroscopic signal. Thus, an experimental diffuse reflectance measurement with a test phantom was performed, using a commercial calibrated photodiode as reference (Hamamatsu S1336-5BQ).

A liquid homogeneous phantom was created, with an absorber (hemoglobin, $\mathrm{Hb}$ ) concentration of $0.5 \mathrm{mg} / \mathrm{mL}$ and a scatterer (polystyrene $1 \mu \mathrm{m}$ beads, representative of collagen fibers) mass concentration of $0.25 \%$, in order to simulate absorption and scattering properties of a GI tissue.

Figure 4 shows the obtained diffuse reflectance for the test phantom, using the commercial photodiode and the fabricated photodiode with $100 \times 100 \mu \mathrm{m}^{2}$.

As it can be observed, the obtained spectra are very similar, which proves de viability of using the fabricated $n+/ p$-epilayer photodiode with $100 \times 100 \mu \mathrm{m}^{2}$ in the extraction of the diffuse reflectance signal to early detection of gastrointestinal cancer.

Moreover, concerning the spectra shape, they are in accordance with what was expected, showing the presence of the strongest absorption $\mathrm{Hb}$ peak, close to $410 \mathrm{~nm}$ (the Soret band (17)).

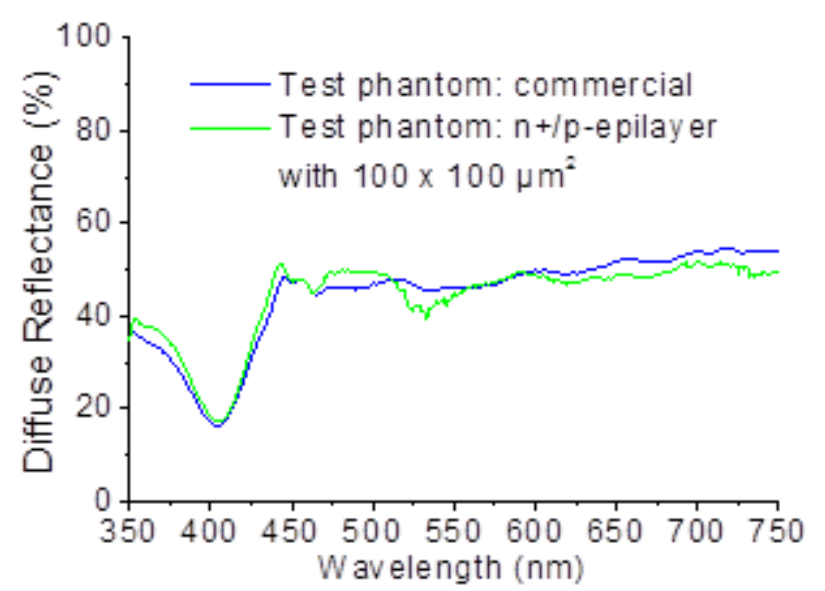

Fig. 4. Diffuse reflectance spectra for a test phantom using a commercial photodiode and the fabricated $\mathrm{n}+/ \mathrm{p}$-epilayer with $100 \times 100 \mu \mathrm{m}^{2}$.

\section{CONCLUSIONS AND FUTURE GUIDELINES}

With this study, we concluded that it is possible to use a silicon photodiode, fabricated with a conventional $0.7 \mu \mathrm{m}$ CMOS process, with $100 \times 100 \mu \mathrm{m}^{2}$ (minimum dimensions) to extract a spectroscopic signal, specifically the diffuse reflectance signal.

Similar tests will be performed with the extraction of the fluorescence signal, especially to verify its capacity to extract the fluorescence signal below $450 \mathrm{~nm}$, due to the low photodiode quantum efficiency (lower than $20 \%$ ).

If not possible, it will be investigated the viability of using avalanche photodiodes with small dimensions to incorporate the photodiodes matrix, in order to improve quantum efficiency between 350 and $450 \mathrm{~nm}$. Some authors reported a technique that allows the fabrication of UV/blue selective avalanche photodiodes in a conventional CMOS process (18).

After that, it will be possible to perform the layout of the photodiodes array and their readout electronics, which will integrate the final microsystem for GI dysplasia detection.

The design and fabrication of 16 optical filters centered in the specific spectral bands (previously considered relevant to GI dysplasia detection) is ongoing in order to connect them on the top of silicon photodiodes, selecting the relevant spectral bands to extract the spectroscopic signals.

\section{ACKNOWLEDGMENT}

This work is funded by FEDER funds through the "Eixo I do Programa Operacional Fatores de Competitividade (POFC) QREN, project references COMPETE: FCOMP-01-0124FEDER-020241 and by FCT - Fundação para a Ciência e a Tecnologia, project reference PTDC/EBB-EBI/120334/2010. S. Pimenta thanks the FCT for the SFRH/BD/87605/2012 PhD grant. R.G. Correia thanks the FCT for the SFRH/BD/70467/2010 PhD grant. 


\section{REFERENCES}

[1] Organization WH. Cancer: Fact sheet №297. November, 2014 [cited 2015 January, 14]. Available from: http://www.who.int/mediacentre/factsheets/fs297/en/.

[2] Yu C-C, Lau C, O'Donoghue G, Mirkovic J, McGee S, Galindo L, et al. Quantitative spectroscopic imaging for non-invasive early cancer detection. Optics express. 2008;16:16227-39.

[3] Georgakoudi I. The color of cancer. Journal of luminescence. 2006;119:75-83

[4] Lo JY, Yu B, Fu HL, Bender JE, Palmer GM, Kuech TF, et al. A strategy for quantitative spectral imaging of tissue absorption and scattering using light emitting diodes and photodiodes. Optics express. 2009;17:1372-84.

[5] Brown J, Vishwanath K, Palmer GM, Ramanujam N. Advances in quantitative UV-visible spectroscopy for clinical and pre-clinical application in cancer. Current opinion in biotechnology. 2009;20:119-31.

[6] Ell C. Improving endoscopic resolution and sampling: fluorescence techniques. Gut. 2003;52:iv30-iv33.

[7] Georgakoudi I, Jacobson BC, Van Dam J, Backman V, Wallace MB Müller MG, et al. Fluorescence, reflectance, and light-scattering spectroscopy for evaluating dysplasia in patients with Barrett's esophagus. Gastroenterology. 2001;120:1620-9.

[8] Mayinger B, Jordan M, Horner P, Gerlach C, Muehldorfer S, Bittorf BR, et al. Endoscopic light-induced autofluorescence spectroscopy for the diagnosis of colorectal cancer and adenoma. Journal of Photochemistry and Photobiology B: Biology. 2003;70:13-20.

[9] Liu N, Chen G, Wu S, Chen R. Distinguishing human normal or cancerous esophagus tissue ex vivo using multiphoton microscopy. Journal of Optics. 2014;16:025301.

[10] Yu B, Lo JY, Kuech TF, Palmer GM, Bender JE, Ramanujam N. Costeffective diffuse reflectance spectroscopy device for quantifying tissue absorption and scattering in vivo. J Biomed Opt. 2008;13:060505.
[11] Ferreira D, Pinto V, Correia JH, Minas G. Spectroscopic detection of gastrointestinal dysplasia using optical microsensors. IEEE Transactions on Biomedical Engineering. 2011;58:2633-9.

[12] Ferreira DS, Mirkovic J, Wolffenbuttel RF, Correia JH, Feld MS, Minas G. Narrow-band pass filter array for integrated opto-electronic spectroscopy detectors to assess esophageal tissue. Biomedical optics express. 2011;2:1703-16.

[13] Pimenta S, Castanheira E, Minas G. Optical Microsystem for Analysis of Diffuse Reflectance and Fluorescence Signals Applied to Early Gastrointestinal Cancer Detection. Sensors. 2015;15:3138-53. PubMed PMID: doi:10.3390/s150203138.

[14] Pimenta S, Castanheira E, Minas G. Preliminary Monte Carlo based inverse model to extract optical tissue properties from experimental diffuse reflectance measurements: coefficients extraction for gastrointestinal dysplasia detection. Photoptics 2014; Lisbon: Proceedings of 2nd International Conference on Photonics, Optics and Laser Technology.

[15] Fernandes A, Cardoso VF, Rocha J, Cabral J, Minas G. Smart-optical detector CMOS array for biochemical parameters analysis in physiological fluids. IEEE Transactions on Industrial Electronics, 2008;55:3192-200.

[16] Vivien L, Rouvière M, Fédéli J-M, Marris-Morini D, Damlencourt JF, Mangeney $J$, et al. High speed and high responsivity germanium photodetector integrated in a Silicon-On-Insulator microwaveguide. Optics Express. 2007; 15:9843-8.

[17] Finlay JC, Foster TH. Effect of pigment packaging on diffuse reflectance spectroscopy of samples containing red blood cells. Optics letters. 2004;29:965-7.

[18] Rochas A, Pauchard AR, Besse P-A, Pantic D, Prijic Z, Popovic RS. Low-noise silicon avalanche photodiodes fabricated in conventional CMOS technologies. IEEE Transactions on Electron Devices. 2002;49:387-394. 